\title{
Knockdown of EIF5A2 inhibits the malignant potential of non-small cell lung cancer cells
}

\author{
CHENG CHEN $^{1 *}$, BOJIA ZHANG ${ }^{2 *}$, SHANSHAN WU ${ }^{3}$, YONGXIANG SONG ${ }^{1}$ and JIAN LI ${ }^{1}$ \\ ${ }^{1}$ Department of Thoracic Surgery, Affiliated Hospital of Zunyi Medical College, Zunyi, Guizhou 563000; \\ ${ }^{2}$ Department of Infectious Disease, Shanghai 10th People's Hospital, Tongji University School of Medicine, \\ Shanghai 200072; ${ }^{3}$ Department of Nursing, Affiliated Hospital of Zunyi Medical College, \\ Zunyi, Guizhou 563000, P.R. China
}

Received March 16, 2016; Accepted February 23, 2017

DOI: $10.3892 / \mathrm{ol} .2018 .7832$

\begin{abstract}
Eukaryotic translation initiation factor $5 \mathrm{~A} 2$ (EIF5A2) has been demonstrated to be upregulated in numerous types of human cancer and is associated with cancer progression. However, the expression and role of EIF5A2 in non-small cell lung cancer (NSCLC) remains unclear. In the present study, the role of EIF5A2 in NSCLC was investigated, in addition to the underlying molecular mechanisms by which EIF5A2 acts. Relative EIF5A2 expression levels were determined in NSCLC cells and compared with levels in non-cancerous lung tissues. Short interfering (si)RNA targeted against EIF5A2 was used to knock down EIF5A2 levels in NSCLC cells. Cell proliferation, apoptosis rate, migration ability and invasion ability were determined in untreated and siRNA-treated NSCLC cells, in addition to the relative protein expression levels of various tumorigenic proteins and E-cadherin. EIF5A2 expression was significantly higher in NSCLC tissues compared with adjacent normal tissues. Knockdown of EIF5A2 in the NSCLC cells significantly inhibited cell proliferation and induced apoptosis. Furthermore, EIF5A2 silencing suppressed cell migratory and invasive capacities in vitro. Silencing of EIF5A2 in the NSCLC cells resulted in the downregulation of the tumorigenic proteins, apoptosis regulator $\mathrm{Bcl}-2$ and myc proto-oncogene protein, and upregulation of E-cadherin, suggesting that EIF5A2 promotes proliferation and metastasis
\end{abstract}

Correspondence to: Dr Jian Li or Dr Yongxiang Song, Department of Thoracic Surgery, Affiliated Hospital of Zunyi Medical College, 201 Dalian Road, Zunyi, Guizhou 563000, P.R. China

E-mail: zyyxylijian@163.com

E-mail: zyyxysyx@163.com

*Contributed equally

Key words: eukaryotic translation initiation factor 5A2, non-small cell lung cancer, epithelial-mesenchymal transition, knockdown through these proteins. EIF5A2 may therefore serve as a novel therapeutic target for the treatment of NSCLC.

\section{Introduction}

Non-small cell lung cancer (NSCLC) accounts for $85 \%$ of pulmonary malignancies (1) and is one of the leading causes of cancer-associated mortality worldwide. Recurrent and metastatic disease is the main cause of mortality, and the 5-year survival rate for lung cancer is $15 \%$ (2). Therefore, in order to improve the prognosis for patients with NSCLC, it is important to understand the underlying molecular mechanisms that contribute to tumor recurrence and metastases, and to identify the relevant markers and targets for treatments.

The epithelial-mesenchymal transition (EMT) is a complex but reversible process whereby epithelial cells acquire a mesenchymal phenotype (3). EMT leads to the loss of the epithelial cell marker E-cadherin, which is a cell-cell junction protein. Loss of E-cadherin is associated with NSCLC progression and metastasis, and with a poor prognosis for patients with NSCLC (4). Therefore, determining an effective method to inhibit EMT in NSCLC may significantly improve the treatment of NSCLC.

Eukaryotic translation initiation factor 5A (EIF5A) is a protein involved in numerous intracellular processes. EIF5A has been demonstrated to serve a role in translation initiation, translation elongation, transcription, mRNA turnover and nucleocytoplasmic transport (5). EIF5A arises in two forms (6,7); EIF5A1 serves an essential role in non-malignant cells, although it has also been implicated in cancer (8). EIF5A2 is a purported oncogene that is less widely expressed in normal tissue $(5,9,10)$; however, EIF5A2 expression has been associated with a number of cancer types, including lung cancer. Downregulation of EIF5A2 prevents EMT in NSCLC (11), while overexpression of EIF5A2 is an adverse prognostic marker of survival for patients with stage I NSCLC (12). In addition, it has been demonstrated that inhibition of EIF5A2 enhances NSCLC sensitivity to chemotherapeutics, prevents or reverses EMT, and reduces the migration and invasion capabilities of NSCLC cells (13).

The role of EIF5A2 as an oncogene has been well established in a number of types of cancer, including NSCLC. 
However, its role in NSCLC cells is not clear. In the present study, the role of EIF5A2 in NSCLC was investigated.

\section{Materials and methods}

Patients and specimens. A total of 47 paired tumor tissue samples and adjacent normal tissues were obtained from primary lung cancer patients undergoing surgical treatment between May 2008 and December 2010 at the Affiliated Hospital of Zunyi Medical College (Zunyi, China). Among the 47 patients with NSCLC, there were 25 males and 22 females aged between 47 and 71 years old, with a mean age of 60 years old. None of the patients had received adjuvant therapy prior to surgery. Following removal, the tissue specimens were immediately fixed in $4 \%$ formaldehyde and embedded in paraffin for immunohistochemical staining. All the tissues were processed within 15 min of removal. Each sample was frozen and stored at $-80^{\circ} \mathrm{C}$. The tumor tissue samples were confirmed by pathological diagnosis, while paired non-cancerous adjacent tisssues were dissected at least $2 \mathrm{~cm}$ away from the tumor border and were confirmed to lack tumor cells by microscopy. The patient clinicopathological features are presented in Table I. The present study was approved by the Ethics Committee of the Affiliated Hospital of Zunyi Medical College, and written informed consent was obtained from each patient enrolled in the study.

Immunohistochemical staining of EIF5A2 expression. Immunohistochemical staining of EIF5A2 was performed to assess relative EIF5A2 expression in NSCLC tissue compared with adjacent normal tissue, according to a standard immunoperoxidase staining protocol. EIF5A2 immunohistochemistry was performed on 4- $\mu \mathrm{m}$ thick, formalin-fixed, paraffin-embedded tissue sections. A rabbit polyclonal antibody anti-EIF5A2 (1:100; cat. no. GTX110510; GeneTex Inc., Irvine, CA, USA) was used for staining (14) and incubated at $37^{\circ} \mathrm{C}$ for $2 \mathrm{~h}$, followed by incubation $1 \mathrm{~h}$ with horseradish peroxidase-conjugated goat anti-rabbit IgG (1:500; cat. no. ab7090; Abcam, Cambridge, MA, USA), then 3,3'-diaminobenzidine (DAB) staining solution (1:25, cat. no. 070004-D; Beijing CellChip Biotechnology Co., Ltd., Beijing, China) for $10 \mathrm{~min}$ at room temperature. The immunohistochemical staining was evaluated using a semi-quantitative score method as follows: $0,0 \% ; 1,<5 \% ; 2$, $\sim 5-50 \%$; and $3,>50 \%$ stained cells. In addition, the staining intensity was scored as $0(-), 1(+), 2(++)$ or $3(+++)$. The final score was defined as the sum of the two parameters, and the samples were grouped as negative (0), weak (1-2), moderate (3) and strong (4-6) staining. Negative and weak staining were defined as low expression of EIF5A2, and moderate and strong staining were defined as high expression of EIF5A2. Only the final immunoreaction scores of the moderate and strong groups were considered as positive for expression. The EIF5A2 expression results are presented in Table I.

Cell culture and transfection. The human bronchial epithelial HBE cell line and five NSCLC cell lines (A549, H23, Calu-3, H1299 and H460) were purchased from the Cell Bank of Type Culture Collection of Chinese Academy of Sciences (Shanghai, China). Cells were cultured in RPMI-1640 medium (Gibco; Thermo Fisher Scientific, Inc., Waltham, MA, USA), supplemented with $100 \mathrm{U} / \mathrm{ml}$ penicillin, $100 \mathrm{mg} / \mathrm{ml}$ streptomycin (Sigma-Aldrich; Merck KGaA, Darmstadt, Germany) and $10 \%$ fetal calf serum (Gibco, Thermo Fisher Scientific, Inc.). Cells were maintained at $37^{\circ} \mathrm{C}$ with $5 \% \mathrm{CO}_{2}$ in a humidified incubator.

Knockdown of EIF5A2 gene expression in NSCLC cells was accomplished using small interfering (si)RNAs targeted against EIF5A2, which were designed and synthesized by Shanghai GenePharma Co., Ltd. (Shanghai, China). The sequence of EIF5A2 siRNA was as follows: 5'-GGAUCU UAAACUGCCAGAATT-3', 5'-UUCUGGCAGUUUAAG AUCCTT-3'. EIF5A2 siRNA (siRNA1, $5 \mu \mathrm{mol} / \mathrm{ml}$; siRNA2, $10 \mu \mathrm{mol} / \mathrm{ml}$; siRNA3, $15 \mu \mathrm{mol} / \mathrm{ml}$ ) or negative control siRNA (5'-UUCUCCGAACGUGUCACGUTT-3'; 5'-ACGUGACAC GUUCGGAGAATT-3') was transfected into the H1299 and H460 cells using Lipofectamine ${ }^{\circledR} 2000$ (Invitrogen; Thermo Fisher Scientific, Inc.) according to the manufacturer's protocol. The transfection medium was replaced with culture medium $6 \mathrm{~h}$ after transfection. All subsequent experiments were performed $24 \mathrm{~h}$ after transfection and repeated three times.

Cell proliferation assay. The cell proliferation assay was performed using Cell Counting Kit-8 (CCK-8; Dojindo Molecular Technologies, Inc., Kumamoto, Japan), according to the manufacturer's protocol. The H1299 and H460 cells treated with EIF5A2 siRNA or control siRNA were seeded on a 96-well microplate at a density of $5 \times 10^{3}$ cells/well. Cell proliferation was assessed at 24,48 and $72 \mathrm{~h}$. CCK- 8 solution $(10 \mu \mathrm{l})$ was added to each well, and the cells were incubated at $37^{\circ} \mathrm{C}$ for an additional $3 \mathrm{~h}$. Optical density (OD) was determined at a wavelength of $450 \mathrm{~nm}$ using an MRX TC II microplate reader (Dynex Technologies, Chantilly, VA, USA).

Cell migration and invasion assays. For the migration assay, H1299 and H460 cells $\left(2 \times 10^{5}\right)$ were resuspended in $100 \mu \mathrm{l}$ serum-free RPMI medium and loaded into the upper chamber of a modified Boyden chamber (Neuro Probe, Inc., Gaithersburg, MD, USA). The lower chamber was filled with RPMI medium and vascular endothelial growth factor $(50 \mathrm{ng} / \mathrm{ml})$. The chamber was incubated at $37^{\circ} \mathrm{C}$ for $24 \mathrm{~h}$. The lower side of the filter was washed with PBS and fixed with $4 \%$ paraformaldehyde. The cells were stained with hematoxylin and counted in three random high-power fields in each well under an Olympus inverted microscope (IX70).

For the invasion assay, the cells $\left(2 \times 10^{5}\right)$ were resuspended in $100 \mu \mathrm{l}$ of serum-free RPMI medium and placed into the upper chamber, which contained an $8-\mu \mathrm{m}$ microporous membrane insert (Costar; Corning Inc., Corning, NY, USA) coated with Matrigel $^{\circledR}$ (BD Biosciences, Franklin Lakes, NJ, USA). The lower chamber contained 10\% FBS plus RPMI-1640 medium. The chamber was incubated at $37^{\circ} \mathrm{C}$ for $24 \mathrm{~h}$. Following this, the lower side of the filter was washed with PBS and fixed with $4 \%$ paraformaldehyde at $37^{\circ} \mathrm{C}$ for $30 \mathrm{~min}$. The cells were stained with hematoxylin at $37^{\circ} \mathrm{C}$ for $15 \mathrm{~min}$ and eosin at $37^{\circ} \mathrm{C}$ for $5 \mathrm{~min}$. The invading cells were imaged and counted using an inverted phase contrast microscope.

Flow cytometry. The apoptosis of cells was assessed using flow cytometry. H1299 and H460 cells were respectively 
Table I. Clinicopathological characteristics of patients with non-small cell lung cancer.

\begin{tabular}{|c|c|c|c|}
\hline \multirow[b]{2}{*}{ Clinicopathological characteristic } & \multicolumn{2}{|c|}{ EIF5A2 expression level } & \multirow[b]{2}{*}{ P-value } \\
\hline & Low $(n=21)$ & High $(n=26)$ & \\
\hline Sex & & & 1.0000 \\
\hline Female & 9 & 11 & \\
\hline Male & 12 & 15 & \\
\hline Age, years & & & 0.5506 \\
\hline$\leq 60$ & 7 & 12 & \\
\hline$>60$ & 14 & 14 & \\
\hline Location of tumor & & & 0.3806 \\
\hline Left & 8 & 14 & \\
\hline Right & 13 & 12 & \\
\hline Histology & & & 0.3881 \\
\hline Adenocarcinoma & 10 & 16 & \\
\hline Squamous cell carcinoma & 11 & 10 & \\
\hline Tumor size, $\mathrm{cm}$ & & & $0.0175^{\mathrm{a}}$ \\
\hline$\leq 3$ & 16 & 10 & \\
\hline$>3$ & 5 & 16 & \\
\hline Lymph node metastasis & & & $0.0200^{\mathrm{a}}$ \\
\hline Yes & 7 & 18 & \\
\hline No & 14 & 8 & \\
\hline Clinical stage & & & 0.7674 \\
\hline I-II & 13 & 14 & \\
\hline III & 8 & 12 & \\
\hline
\end{tabular}

${ }^{\mathrm{a}} \mathrm{P}<0.05$.

plated in 6-well plates at a density of $5 \times 10^{5}$ cells/well. Cells were harvested and fixed with cold $75 \%$ ethanol at $4^{\circ} \mathrm{C}$ overnight. The fixed cells were collected and labeled with $10 \mu \mathrm{g} / \mathrm{ml}$ anti-Annexin V-fluorescein isothiocyanate (FITC; eBioscience, Inc., San Diego, CA, USA) and propidium iodide (PI; eBioscience, Inc.) at $4^{\circ} \mathrm{C}$ for $1 \mathrm{~h}$. The degree of apoptosis was expressed as the percentage of cells stained with Annexin V-FITC/PI and analyzed by fluorescence activated cell sorting using a FACscan flow cytometer (BD Biosciences FACScan) and WinList software (version 7.0; Verity Software House, Inc., Topsham, ME, USA).

Western blot analysis. The non-cancerous human bronchial epithelial cell line, in addition to the NSCLC A549, H23, Calu-3, H1299 and H460 cell lines, were resuspended in $300 \mu 1$ cell lysis buffer (Cell Signaling Technology, Inc., Danvers, MA, USA) with $1 \mathrm{X}$ protease inhibitors and $1 \mathrm{mM}$ phenylmethanesulfonyl fluoride (Sigma-Aldrich; Merck KGaA). The protein concentration was quantified using a bicinchoninic acid protein assay kit (Thermo Fisher Scientific, Inc.). A total of $40 \mu \mathrm{g}$ of protein was loaded in each well and run on a $10 \%$ SDS-PAGE gel, and the proteins were transferred to polyvinylidene fluoride membranes (Merck KGaA). The membranes were blocked with Tris-buffered saline (TBS) and with TBS containing $0.1 \%$ Tween-20 and $5 \%$ bovine serum albumin at $4^{\circ} \mathrm{C}$ overnight. Western blotting was performed using primary antibodies against EIF5A2 (dilution, 1:500; cat. no. 17069-1-AP; ProteinTech Group, Inc., Chicago, IL, USA), ww (dilution, 1:5,000 in 5\% milk; cat. no. I-19; Santa Cruz Biotechnology, Inc., Dallas, TX, USA), Vimentin (cat. no. 13614), E-cadherin (cat. no. 5296), Ras-related C3 botulinum toxin substrate 1 (Rac1; cat. no. 2465), apoptosis regulator Bcl-2 (Bcl-2; cat. no. 2872) and myc proto-oncogene protein (c-Myc; cat. no. 9402; all dilutions, 1:1,000; Cell Signaling Technology, USA) and incubated overnight at $4^{\circ} \mathrm{C}$. Subsequently, the secondary goat anti-rabbit antibodies (1:4,000, cat. no. NEF812001EA; PerkinElmer, Inc., Waltham, MA, USA) were added and incubated for $1 \mathrm{~h}$ at room temperature. The blots were then re-probed with horseradish peroxidase-conjugated secondary antibody and visualized using the SuperSignal West Pico chemiluminescent substrate (Pierce; Thermo Fisher Scientific, Inc.). Autoradiographs were scanned using ImageQuant LAS 4010 Imaging System (GE Healthcare, Chicago, IL, USA) and analyzed semi-quantitatively. All images are representative of at least three separate experiments.

Statistical analysis. Quantitative values are presented as the mean \pm standard deviation. Student's t-test was performed to determine statistically significant differences between groups. Statistical analysis was performed using SPSS software (version 16.0; SPSS, Inc., Chicago, IL, USA). P $<0.05$ was considered to indicate a statistically significant difference. 
A

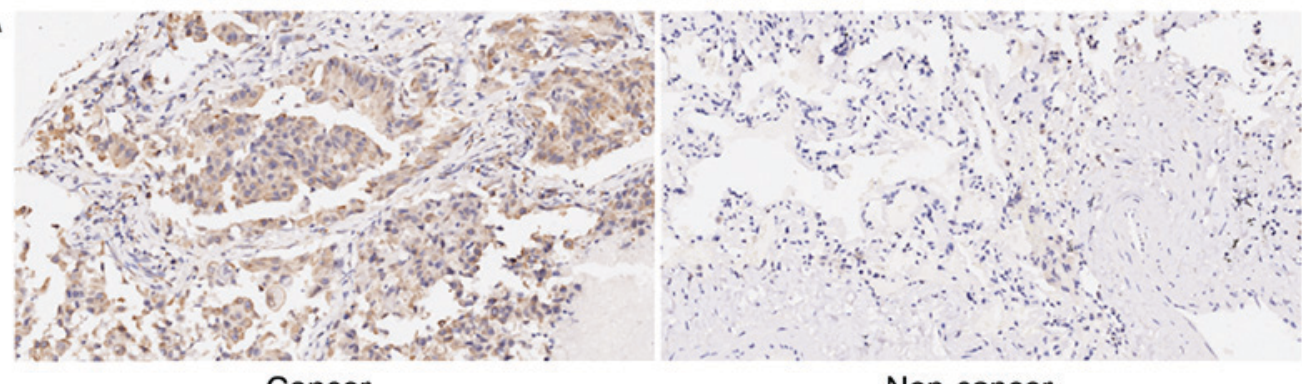

Cancer

Non-cancer

B
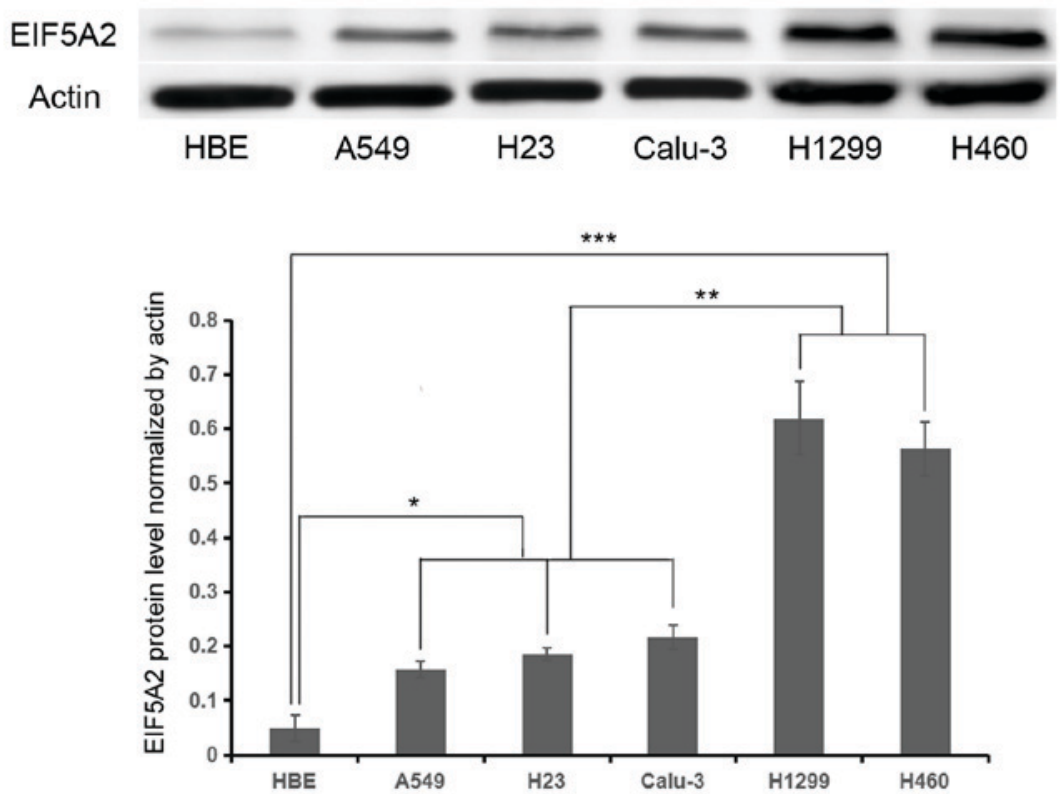

Figure 1. EIF5A2 expression is upregulated in NSCLC tissues and cells. (A) Immunohistochemical staining of EIF5A2 demonstrated increased EIF5A2 expression in NSCLC tissue (left) compared with adjacent non-cancerous tissue (right). Images captures at x200 magnification. (B) Western blot analysis demonstrated increased EIF5A2 expression in NSCLC A549, H23, Calu-3, H1299 and H460 cell lines compared with the human bronchial epithelial HBE cell line. ${ }^{*} \mathrm{P}<0.05,{ }^{* *} \mathrm{P}<0.01,{ }^{* * *} \mathrm{P}<0.001$. EIF5A2, eukaryotic translation initiation factor 5A; NSCLC, non-small cell lung cancer.

\section{Results}

EIF5A2 expression is upregulated in NSCLC cells. To assess whether or not EIF5A2 is upregulated in NSCLC samples and cells, immunohistochemical staining and western blot analysis were performed. Immunohistochemical staining demonstrated stronger EIF5A2 expression (score: Strong, 5-6) in NSCLC tissue compared with adjacent benign lung tissue (score: Negative or weak, 0-1) (Fig. 1A). A total of 26 patients with NSCLC (>50\%) exhibited high expression of EIF5A2. Western blot analysis demonstrated that the relative EIF5A2 expression normalized by actin were $0.05 \pm 0.03,0.16 \pm 0.02$, $0.19 \pm 0.01,0.22 \pm 0.02,0.62 \pm 0.07,0.56 \pm 0.05$ in the HBE, A549, H23, Calu-3, H1299 and H460 cell lines, respectively. Compared with the benign human bronchial epithelial HBE cell line, increased EIF5A2 expression was observed in all NSCLC lines $(\mathrm{P}<0.05)$, and H1299 and H460 demonstrated higher EIF5A2 expression compared with in other NSCLC lines $(\mathrm{P}<0.01$; Fig. 1B).

EIF5A2 silencing inhibits cell growth and induces apoptosis of NSCLC cells in vitro. EIF5A2 siRNAs (siRNA1, $5 \mu \mathrm{mol} / \mathrm{ml}$; siRNA2, $10 \mu \mathrm{mol} / \mathrm{ml}$; siRNA3, $15 \mu \mathrm{mol} / \mathrm{ml}$ ) were separately transfected into H1299 or H460 cells in order to silence EIF5A2 expression. The silencing efficacy of three concentrations of EIF5A2 siRNA was confirmed by western blotting analysis. EIF5A2 protein levels normalized by actin were $0.64 \pm 0.10$, $0.42 \pm 0.06,0.14 \pm 0.02,0.05 \pm 0.01$ in NC, siRNA1, siRNA2 and siRNA3 groups of the H1299 cell line, respectively; whereas, EIF5A2 protein levels were $0.60 \pm 0.04,0.40 \pm 0.04,0.14 \pm 0.02$ and $0.05 \pm 0.01$ in NC, siRNA1, siRNA2 and siRNA3 groups of the H460 cell line, respectively. EIF5A2 expression was significantly inhibited in the H1299 and H460 cell lines treated with EIF5A2 siRNA compared with those treated with control siRNA $(\mathrm{P}<0.05)$, thus confirming the knockdown (Fig. 2A). Following comparison of the knockdown efficiency of three concentration of EIF5A2 siRNA, $10 \mu \mathrm{mol} / \mathrm{ml}$ EIF5A2 siRNA was chosen for further study.

Cell proliferation was then assessed in H1299 and H460 cells treated with EIF5A2 siRNA and compared with cells treated with control siRNA. The OD values for H1299 cells treated with EIF5A2 siRNA were $0.23 \pm 0.03,0.47 \pm 0.06$, $0.87 \pm 0.08$ and $1.47 \pm 0.15$ at $0,1,2$ and 3 days, respectively, while the OD values for $\mathrm{H} 1299$ cells treated with control siRNA were $0.23 \pm 0.04,0.42 \pm 0.06,0.67 \pm 0.05$ and $1.04 \pm 0.15$. For H460 cells treated with EIF5A2 siRNA, the OD values were $0.23 \pm 0.04,0.45 \pm 0.04,0.82 \pm 0.07$ and $1.50 \pm 0.10$ at $0,1,2$ and 3 days, respectively, while for control siRNA transfected 
A
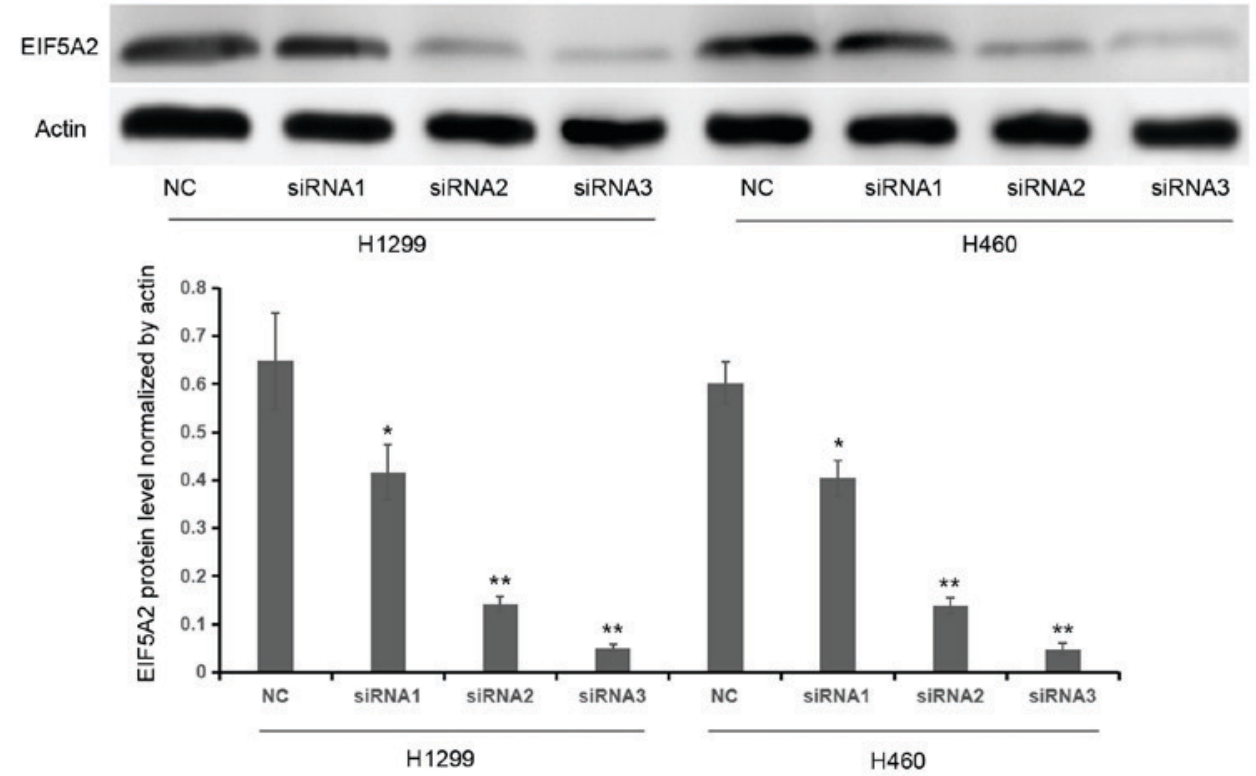

B
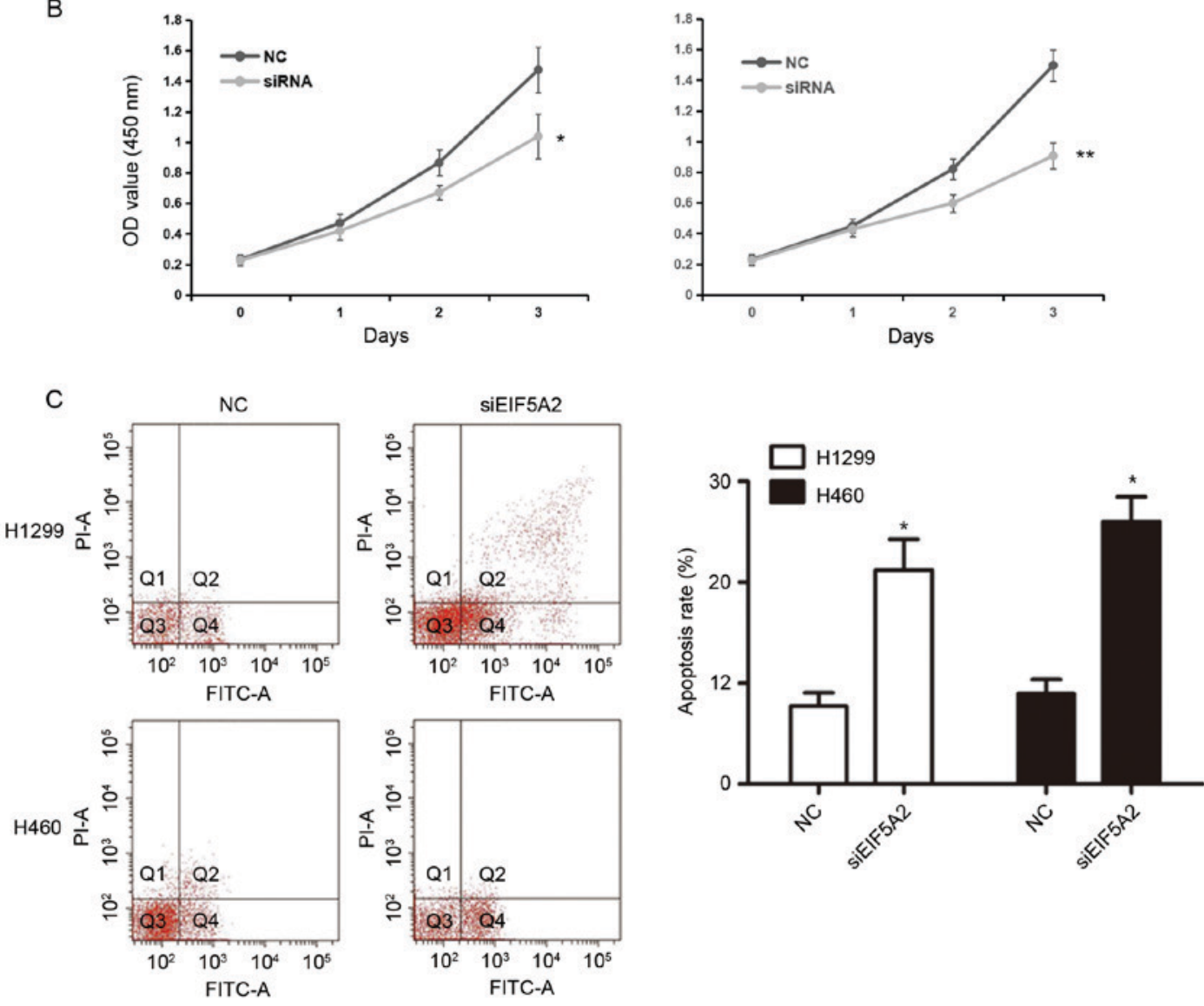

Figure 2. EIF5A2 silencing inhibits cell growth and induces apoptosis of non-small cell lung cancer cells in vitro. (A) Western blot analysis of EIF5A2 expression in H1299 and H460 cells transfected with EIF5A2 siRNA (siRNA1, siRNA2 and siRNA3) or control siRNA. $\beta$-actin was used as the loading control. ${ }^{*} \mathrm{P}<0.05,{ }^{* *} \mathrm{P}<0.01$. (B) Cell proliferation assay of H1299 $\left({ }^{*} \mathrm{P}<0.05\right)$ and $\mathrm{H} 460\left({ }^{* * *} \mathrm{P}<0.01\right)$ cells in which EIF5A2 was suppressed (siEIF5A2) demonstrated significantly lower rates of cell proliferation than in the control. (C) Flow cytometry results demonstrated significantly higher rates of apoptosis in H1299 and H460 cells treated with the EIF5A2 siRNA (siEIF5A2) compared with cells treated with the control siRNA ("P<0.05). EIF5A2, eukaryotic translation initiation factor 5A; NC, control; siRNA, small interfering RNA; OD, optical density; FITC-A, anti-Annexin V-fluorescein isothiocyanate; PI, propidium iodide.

cells, the OD values were $0.22 \pm 0.03,0.43 \pm 0.05,0.60 \pm 0.06$ and $0.91 \pm 0.08$, respectively. Cells treated with EIF5A2 siRNA exhibited significantly reduced cellular proliferation 3 days after transfection compared with the cells treated with control siRNA (H1299, P<0.05; H460, P<0.01; Fig. 2B).
The apoptosis rate of cells following transfection was determined by flow cytometry. At $72 \mathrm{~h}$ post-siRNA transfection, the apoptosis rates of the H1299 and H460 cells transfected with EIF5A2 siRNA were significantly higher compared with those of H1299 and H460 cells treated with control siRNA 

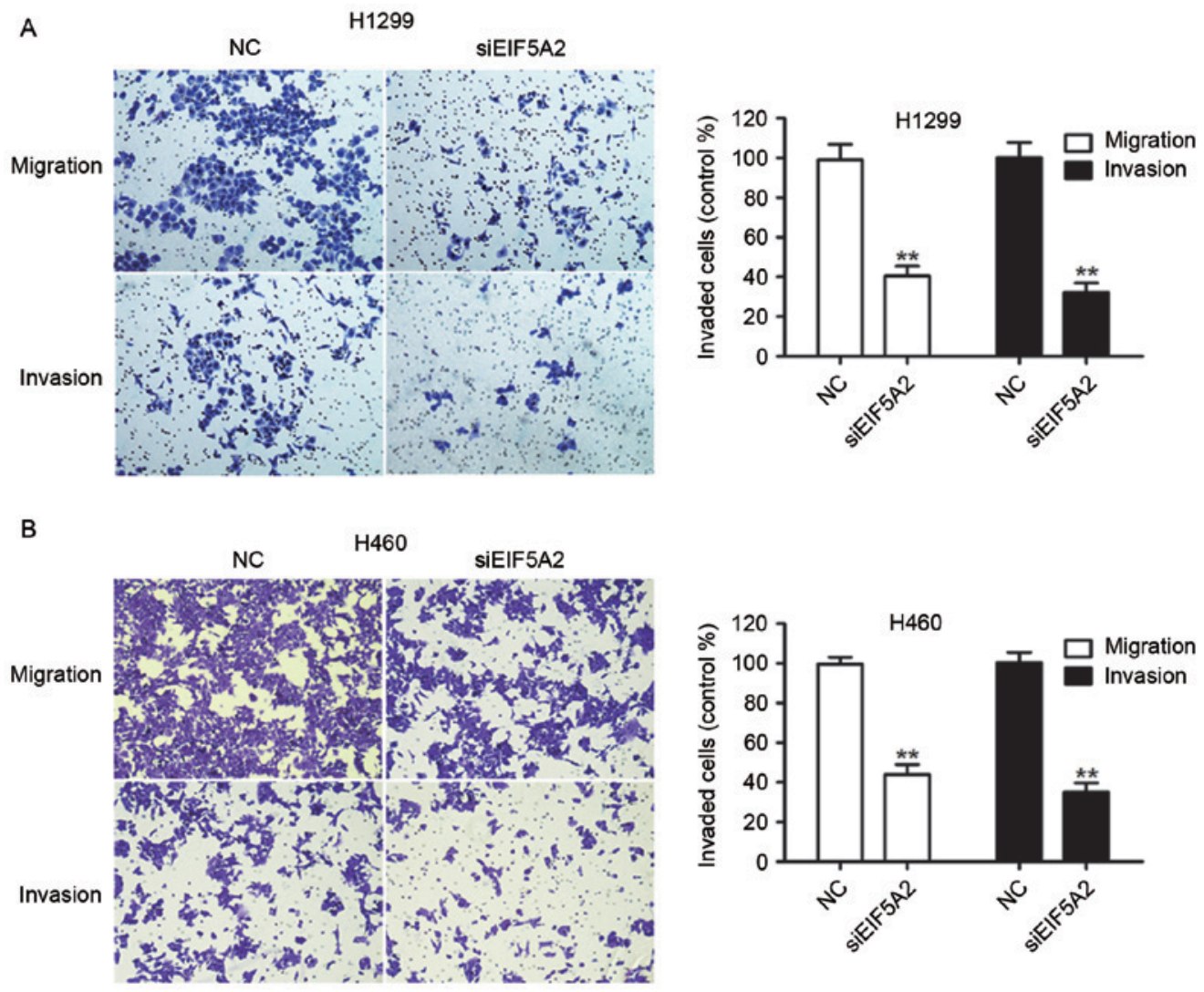

Figure 3. Knockdown of EIF5A2 inhibits NSCLC cell migration and invasion in vitro. (A) H1299 cells in which EIF5A2 was suppressed (siEIF5A2) exhibit significantly lower rates of migration (upper panel) and invasion (lower panel) than the control ("* $\mathrm{P}<0.01)$. (B) H460 cells treated with EIF5A2 siRNA (siEIF5A2) exhibited significantly lower rates of migration (upper panel) and invasion (lower panel) than the control ("P<0.01). EIF5A2, eukaryotic translation initiation factor 5A; NSCLC, non-small cell lung cancer; NC, control; siRNA, small interfering RNA.

$(21.22 \pm 3.05$ and $26.03 \pm 2.43$ vs. $7.71 \pm 1.34$ and $8.96 \pm 1.41$ respectively; P<0.05; Fig. 2C).

Knockdown of EIF5A2 inhibits NSCLC cell migration and invasion in vitro. Migration and invasion assays were performed to assess the effect of EIF5A2 silencing on the metastatic capacity of NSCLC cells. H1299 cells treated with EIF5A2 siRNA exhibited a significantly decreased ability to migrate and invade compared with H1299 cells treated with control siRNA $(40.43 \pm 4.98$ vs. $97.00 \pm 4.37 \%$; $\mathrm{P}<0.01)$ and $(32.19 \pm 4.75$ vs. $98.12 \pm 5.06 \%, \mathrm{P}<0.01)$, respectively (Fig. 3A). Similarly, H460 cells treated with EIF5A2 siRNA demonstrated a significantly decreased ability to migrate and invade compared with $\mathrm{H} 460$ cells treated with control siRNA $(40.58 \pm 4.38$ vs. $99.70 \pm 3.46 \%, P<0.01$; Fig. $3 \mathrm{~B})$ and $(34.04 \pm 3.26$ vs. $100.43 \pm 5.11 \%, \mathrm{P}<0.01)$.

EIF5A2 regulates the expression of $c-M y c, B c l-2, R a c l$ and $E$-cadherin in NSCLC cells. To investigate the underlying molecular mechanism of the effect of EIF5A2 on cell proliferation, apoptosis rate and metastatic capacity, expression levels of various proteins were measured. Western blot analysis revealed that the protein expression levels of Vimentin, E-cadherin, Rac1, Bcl-2 and c-Myc normalized to actin were $0.92 \pm 0.02,0.03 \pm 0.02,0.62 \pm 0.22,0.42 \pm 0.22$ and $0.49 \pm 0.20$, respectively, in the control group of $\mathrm{H} 1299$ cell line; whereas, in the siEIF5A2 group they were $0.2 \pm 0.08,0.17 \pm 0.05,0.22 \pm 0.03$, $0.22 \pm 0.02$ and $0.20 \pm 0.04$, respectively. In the $\mathrm{H} 460$ cell line, the protein expression levels of Vimentin, E-cadherin, Rac1, Bcl-2 and c-Myc normalized to actin were $0.96 \pm 0.02,0.10 \pm 0.02$, $0.47 \pm 0.06,0.24 \pm 0.01$ and $0.86 \pm 0.04$, respectively, in control group; whereas, in the siEIF5A2 group they were $0.34 \pm 0.10$, $0.23 \pm 0.03,0.16 \pm 0.04,0.14 \pm 0.01$ and $0.20 \pm 0.07$, respectively. These results demonstrated that $\mathrm{H} 1299$ and $\mathrm{H} 460$ cells treated with EIF5A2 siRNA expressed lower levels of Vimentin, Rac1, Bcl-2 and c-Myc, and higher levels of E-cadherin compared with the H1299 and H460 cells treated with control siRNA (P<0.05; Fig. 4).

\section{Discussion}

EIF5A2 has been implicated in a number of cancers, including bladder cancer, colorectal cancer, pancreatic adenocarcinoma, esophageal squamous cell carcinoma, gastric adenocarcinoma, breast cancer, prostate adenocarcinoma, hepatocellular carcinoma, ovarian cancer and melanoma $(9,14-22)$. Patients with esophageal squamous cell carcinoma, bladder cancer and pancreatic adenocarcinoma in which EIF5A2 is overexpressed have been demonstrated to exhibit statistically significantly shorter survival times $(14,17,20)$. Furthermore, patients with hepatocellular carcinoma in which EIF5A2 is overexpressed have a greater number of metastases and are more likely to present with venous invasion $(23,24)$. These effects on survival times suggest that EIF5A2 is not only important in malignant transformation, but also may serve as a driver of tumor progression. 


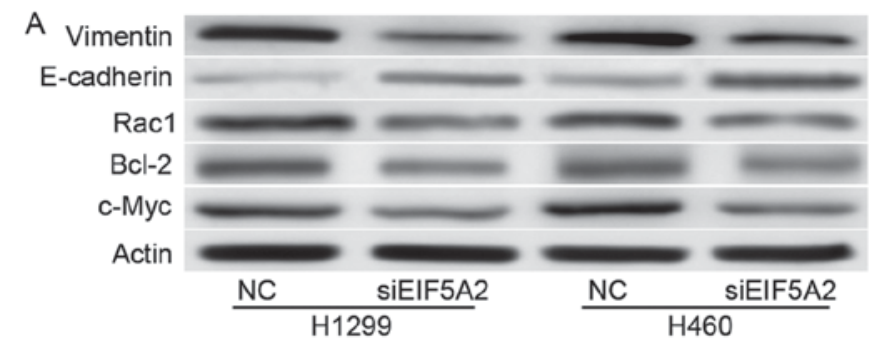

B
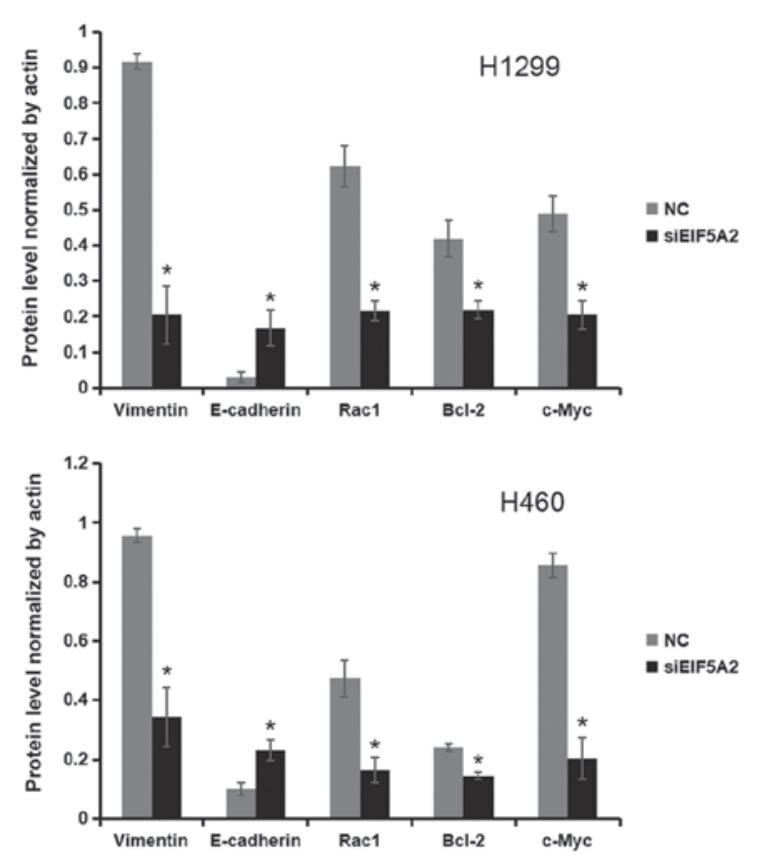

Figure 4. EIF5A2 regulates the expression of c-Myc, Bcl-2, Rac1 and E-cadherin in non-small cell lung cancer cells. (A) Western blot analysis and (B) quantification of the western blots demonstrated reduced expression of Rac1, Bcl-2, and c-Myc, and increased expression of E-cadherin in H1299 and $\mathrm{H} 460$ cells treated with the EIF5A2 siRNA (siEIF5A2) compared with cells treated with control siRNA. Rac1, Ras-related C3 botulinum toxin substrate 1; Bcl-2, apoptosis regulator Bcl-2; c-Myc, myc proto-oncogene protein; EIF5A2, eukaryotic translation initiation factor 5A; NC, control siRNA, small interfering RNA. "P<0.05.

Previous studies have demonstrated that EIF5A2 exhibits its tumorigenic effects via activation of the phosphoinositide-3-kinase/Rac- $\alpha$ serine/threonine-protein kinase signaling pathway $(15,25)$ and its induction of matrix metalloproteinase 2 protein expression (26). In addition, EIF5A2 reduces expression of $\mathrm{E}$-cadherin and increases expression of Vimentin $(26,27)$, and may activate transforming protein RhoA precursor/Racl signaling pathways (24). Additionally, a previous study identified zinc finger protein Glil as a putative transcription factor for EIF5A2 (28).

Data from the present study demonstrated that EIF5A2 was overexpressed in NSCLC cells compared with cells from adjacent non-cancerous lung tissues, which is in agreement with the results of previous studies $(11,13)$. NSCLC cells treated with EIF5A2 siRNA exhibited significantly reduced proliferation, significantly increased rates of apoptosis, and significantly reduced abilities to migrate and invade. In addition, the underlying molecular mechanisms by which EIF5A2 exhibits its effects were investigated. In NSCLC cells treated with EIF5A2 siRNA, the expression of the tumorigenic proteins Rac1, Bcl-2, and c-Myc was decreased compared with that of NSCLC cells treated with control siRNA. Rac1 has been widely implicated in cancer cell invasion and metastasis, Bcl-2 has been demonstrated to be an anti-apoptotic protein that can promote tumorigenesis and Myc overexpression stimulates gene amplification, potentially by stimulating DNA replication (29-31). Furthermore, in NSCLC cells treated with EIF5A2 siRNA in the present study, the anti-malignant protein E-cadherin was overexpressed, and the EMT marker Vimentin was downregulated compared with the expression levels found in the NSCLC cells treated with control siRNA.

The results from the present study suggest that EIF5A2 may serve as a therapeutic target for the treatment of NSCLC, for which an EIF5A2 siRNA may be effective. SiRNA is not widely used due to challenges with successfully delivering the siRNA to the tumor; however, a previous study demonstrated that not only was delivery of EIF5A2 interference RNA to a tumor in vivo possible, but that it also resulted in tumor suppression (14). The aforementioned study examined the effects of EIF5A2 siRNA on bladder cancer, therefore further research is required to confirm that EIF5A2 siRNA could be delivered to NSCLC cells in vivo. Using EIF5A2 siRNA as a treatment is also limited by potential side effects. EIF5A2 silencing in non-target benign tissue could cause unanticipated side effects and such side effects would require thorough investigation prior to the wide use of siRNA as a therapy for NSCLC.

Other types of treatment that inhibit EIF5A2 may prove more technically feasible in terms of their ability to reach a tumor in vivo. MicroRNA-30b (miR-30b) and miR-125b have been demonstrated to suppress gastric adenocarcinoma cells and hepatocellular carcinoma cells respectively via their direct inhibitory effects on EIF5A2 translation $(20,22)$. Therefore, any compound that increases miR-30b and/or miR-125b transcription has the potential to serve as a successful therapy for NSCLC. In addition, the compound N1-guanyl-1,7-diaminoheptane (GC7) is a direct EIF5A2 inhibitor and therefore has the potential to be an effective therapy for NSCLC (17-19,21).

While EIF5A2 inhibitors have the potential to serve as individual targeted chemotherapeutics, a successful therapy regimen may require the inclusion of a mix of targeted and more traditional drugs. Prior studies have demonstrated that EIF5A2 inhibition lowers the chemoresistance of colorectal cancer cells to doxorubicin, breast cancer cells to doxorubicin (18), hepatocellular carcinoma cells to 5-fluorouracil (32), hepatocellular carcinoma cells to doxorubicin (33), bladder cancer cells to doxorubicin (34) and esophageal squamous cell carcinoma cells to 5-fluorouracil, docetaxel and taxol (35). While this effect on chemoresistance would require confirmation in NSCLC cells, these prior results suggest that the role of EIF5A2 inhibitors in NSCLC treatment may be adjunctive as opposed to primary in nature.

Additionally, EIF5A 2 could serve as a marker of disease severity; EIF5A2 has been suggested to be a potential biomarker for esophageal squamous cell carcinoma (24) and melanoma (23). Data from the present study revealed that NSCLC cells that overexpressed EIF5A2 exhibited a greater malignant tendency compared with NSCLC cells that expressed lower levels of EIF5A2. Therefore, the level of EIF5A2 expression in a patient's tumor may predict the aggressiveness of that tumor. In a patient, EIF5A2 levels would have 
to be determined via biopsy, although if the protein is secreted in the blood, serum tests may also prove useful. Patients with aggressive tumors expressing high levels of EIF5A2 could be treated with more aggressive therapeutic strategies, including pneumonectomy or lobectomy with mediastinal lymph node dissection, or prolonged chemotherapeutic regimens. Patients with less aggressive tumors expressing lower levels of EIF5A2 could be treated more conservatively, with less aggressive chemotherapeutics or less extensive resections.

In the present study, five NSCLC cell lines (A549, H23, Calu-3, H1299 and H460) were examined. All the cell lines overexpressed EIF5A2 compared with the non-cancerous human bronchial epithelium HBE cell line; however, H1299 and H460 cells expressed the greatest amount of EIF5A2 compared with the other NSCLC cell lines. Subsequent experiments in the present study were then performed on the H1299 and H460 cell lines only. Therefore, while the present study has demonstrated that silencing of EIF5A2 has an inhibitory effect on the malignant potential of cells from the H1299 and H460 cell lines, this may not be the case for other NSCLC cell lines. Different tumor cells can exhibit different phenotypic characteristics, leading to heterogeneity in tumors, therefore, while EIF5A2 may serve as a potential therapeutic target or marker of disease severity for certain patients with NSCLC's, it may not do so for other patients.

The data from the present study suggest that EIF5A2 serves as a tumorigenic protein in NSCLC primarily by increasing cell proliferation, inhibiting apoptosis and promoting metastasis. Knockdown of EIF5A2 reverses these tumorigenic effects, suggesting that EIF5A2 inhibitors may serve as effective therapies for NSCLC.

\section{References}

1. D'Addario G, Früh M, Reck M, Baumann P, Klepetko W and Felip E; ESMO Guidelines Working Group: Metastatic non-small-cell lung cancer: ESMO clinical practice guidelines for diagnosis, treatment and follow-up. Ann Oncol 21 (Suppl 5): v116-v119, 2010.

2. Rosell R, Felip E, Taron M, Majo J, Mendez P, Sanchez-Ronco M, Queralt C, Sanchez JJ and Maestre J: Gene expression as a predictive marker of outcome in stage IIB-IIIA-IIIB non-small cell lung cancer after induction gemcitabine-based chemotherapy followed by resectional surgery. Clin Cancer Res 10: 4215s-4219s, 2004.

3. Thiery JP and Sleeman JP: Complex networks orchestrate epithelial-mesenchymal transitions. Nat Rev Mol Cell Biol 7: 131-142, 2006.

4. Thiery JP: Epithelial-mesenchymal transitions in tumour progression. Nat Rev Cancer 2: 442-454, 2002.

5. Mathews MB and Hershey JW: The translation factor eIF5A and human cancer. Biochim Biophys Acta 1849: 836-844, 2015.

6. Ishfaq M, Maeta K, Maeda S, Natsume T, Ito A and Yoshida M The role of acetylation in the subcellular localization of an oncogenic isoform of translation factor eIF5A. Biosci Biotechnol Biochem 76: 2165-2167, 2012.

7. Tong Y, Park I, Hong BS, Nedyalkova L, Tempel W and Park HW: Crystal structure of human eIF5A1: Insight into functional similarity of human eIF5A1 and eIF5A2. Proteins 75: 1040-1045, 2009.

8. Fujimura K, Wright T, Strnadel J,Kaushal S, Metildi C,Lowy AM, Bouvet M, Kelber JA and Klemke RL: A hypusine-eIF5A-PEAK1 switch regulates the pathogenesis of pancreatic cancer. Cancer Res 74: 6671-6681, 2014.

9. Clement PM, Henderson CA, Jenkins ZA, Smit-McBride Z, Wolff EC, Hershey JW, Park MH and Johansson HE: Identification and characterization of eukaryotic initiation factor 5A-2. Eur J Biochem 270: 4254-4263, 2003.
10. Jenkins ZA, Hååg PG and Johansson HE: Human eIF5A2 on chromosome 3q25-q27 is a phylogenetically conserved vertebrate variant of eukaryotic translation initiation factor $5 \mathrm{~A}$ with tissue-specific expression. Genomics 71: 101-109, 2001.

11. Xu GD, Shi XB, Sun LB, Zhou QY, Zheng DW, Shi HS, Che YL, Wang ZS and Shao GF: Down-regulation of eIF5A-2 prevents epithelial-mesenchymal transition in non-small-cell lung cancer cells. J Zhejiang Univ Sci B 14: 460-467, 2013.

12. He LR, Zhao HY, Li BK, Liu YH, Liu MZ, Guan XY, Bian XW, Zeng YX and Xie D: Overexpression of eIF5A-2 is an adverse prognostic marker of survival in stage I non-small cell lung cancer patients. Int J Cancer 129: 143-150, 2011.

13. Xu G, Yu H, Shi X, Zhou Q, Zheng D, Shi H, Li N, Zhang X and Shao G: Cisplatin sensitivity is enhanced in non-small cell lung cancer cells by regulating epithelial-mesenchymal transition through inhibition of eukaryotic translation initiation factor 5A2. BMC Pulm Med 14: 174, 2014.

14. Wei YX, Chen G, You L and Zhao YP: Expression of eukaryotic translation initiation factor $5 \mathrm{~A} 2$ in pancreatic adenocarcinoma and its correlation with the prognosis. Zhongguo Yi Xue Ke Xue Yuan Xue Bao 35: 634-638, 2013 (In Chinese).

15. Khosravi S, Wong RP, Ardekani GS, Zhang G, Martinka M, Ong CJ and Li G: Role of EIF5A2, a downstream target of Akt, in promoting melanoma cell invasion. Br J Cancer 110: 399-408, 2014.

16. Li S, Ma Y, Xie C, Wu Z, Kang Z, Fang Z, Su B and Guan M: EphA6 promotes angiogenesis and prostate cancer metastasis and is associated with human prostate cancer progression. Oncotarget 6: 22587-22597, 2015.

17. Li Y, Fu L, Li JB, Qin Y, Zeng TT, Zhou J, Zeng ZL, Chen J, Cao TT, Ban X, et al: Increased expression of EIF5A2, via hypoxia or gene amplification, contributes to metastasis and angiogenesis of esophageal squamous cell carcinoma. Gastroenterology 146: 1701-1713.e9, 2014.

18. Liu Y, Liu R, Fu P, Du F, Hong Y, Yao M, Zhang X and Zheng S: N1-Guanyl-1,7-diaminoheptane sensitizes estrogen receptor negative breast cancer cells to doxorubicin by preventing epithelial-mesenchymal transition through inhibition of eukaryotic translation initiation factor 5A2 activation. Cell Physiol Biochem 36: 2494-2503, 2015.

19. Meng QB, Kang WM, Yu JC, Liu YQ, Ma ZQ, Zhou L, Cui QC and Zhou WX: Overexpression of eukaryotic translation initiation factor 5A2 (EIF5A2) correlates with cell aggressiveness and poor survival in gastric cancer. PLoS One 10: e0119229, 2015.

20. Wei JH, Cao JZ, Zhang D, Liao B, Zhong WM, Lu J, Zhao HW, Zhang JX, Tong ZT, Fan S, et al: EIF5A 2 predicts outcome in localised invasive bladder cancer and promotes bladder cancer cell aggressiveness in vitro and in vivo. Br J Cancer 110: 1767-1777, 2014.

21. Xu X, Liu H, Zhang H, Dai W, Guo C, Xie C, Wei S, He S and $\mathrm{Xu} \mathrm{X}$ : Sonic hedgehog-GLI family zinc finger 1 signaling pathway promotes the growth and migration of pancreatic cancer cells by regulating the transcription of eukaryotic translation initiation factor 5A2. Pancreas 44: 1252-1258, 2015.

22. Zhu W, Cai MY, Tong ZT, Dong SS, Mai SJ, Liao YJ, Bian XW, Lin MC, Kung HF, Zeng YX, et al: Overexpression of EIF5A2 promotes colorectal carcinoma cell aggressiveness by upregulating MTA1 through C-myc to induce epithelialmesenchymaltransition. Gut 61: 562-575, 2012.

23. Shek FH, Fatima S and Lee NP: Implications of the use of eukaryotic translation initiation factor 5A (eIF5A) for prognosis and treatment of hepatocellular carcinoma. Int J Hepatol 2012: 760928, 2012.

24. Tang DJ, Dong SS, Ma NF, Xie D, Chen L, Fu L, Lau SH, Li Y, $\mathrm{Li}$ Y and Guan XY: Overexpression of eukaryotic initiation factor 5A2 enhances cell motility and promotes tumor metastasis in hepatocellular carcinoma. Hepatology 51: 1255-1263, 2010.

25. Chen Z, Yu T, Zhou B, Wei J, Fang Y, Lu J, Guo L, Chen W, Liu ZP and Luo J: Mg(II)-Catechin nanoparticles delivering siRNA targeting EIF5A2 inhibit bladder cancer cell growth in vitro and in vivo. Biomaterials 81: 125-134, 2016.

26. Bao Y, Lu Y, Wang X, Feng W, Sun X, Guo H, Tang C, Zhang X, Shi Q and $\mathrm{Yu} \mathrm{H}$ : Eukaryotic translation initiation factor 5A2 (eIF5A2) regulates chemoresistance in colorectal cancer through epithelial mesenchymal transition. Cancer Cell Int 15: 109, 2015.

27. Tian SB, Yu JC, Liu YQ, Kang WM, Ma ZQ, Ye X and Yan C: MiR-30b suppresses tumor migration and invasion by targeting EIF5A2 in gastric cancer. World J Gastroenterol 21: 9337-9347, 2015. 
28. Tsang FH, Au V, Lu WJ, Shek FH, Liu AM, Luk JM, Fan ST, Poon RT and Lee NP: Prognostic marker microRNA-125b inhibits tumorigenic properties of hepatocellular carcinoma cells via suppressing tumorigenic molecule eIF5A2. Dig Dis Sci 59: 2477-2487, 2014

29. Chen QY, Zheng Y, Jiao DM, Chen FY, Hu HZ, Wu YQ, Song J, Yan J, Wu LJ and Lv GY: Curcumin inhibits lung cancer cell migration and invasion through Rac1-dependent signaling pathway. J Nutr Biochem 25: 177-185, 2014

30. Coultas L and Strasser A: The role of the Bcl-2 protein family in cancer. Semin Cancer Biol 13: 115-123, 2003.

31. Khaleghian M, Shakoori A, Razavi AE and Azimi C: Relationship of amplification and expression of the C-MYC gene with survival among gastric cancer patients. Asian Pac J Cancer Prev 16: 7061-7069, 2015.

32. Wang FW, Cai MY, Mai SJ, Chen JW, Bai HY, Li Y, Liao YJ, Li CP, Tian XP, Kung HF, et al: Ablation of EIF5A2 induces tumor vasculature remodeling and improves tumor response to chemotherapy via regulation of matrix metalloproteinase 2 expression. Oncotarget 5: 6716-6733, 2014.
33. Lou B, Fan J, Wang K, Chen W, Zhou X, Zhang J, Lin S, Lv F and Chen Y: N1-guanyl-1,7-diaminoheptane (GC7) enhances the therapeutic efficacy of doxorubicin by inhibiting activation of eukaryotic translation initiation factor 5A2 (eIF5A2) and preventing the epithelial-mesenchymal transition in hepatocellular carcinoma cells. Exp Cell Res 319: 2708-2717, 2013.

34. Yang J, Yu H, Shen M, Wei W, Xia L and Zhao P: N1-guanyl1,7-diaminoheptane sensitizes bladder cancer cells to doxorubicin by preventing epithelial-mesenchymal transition through inhibition of eukaryotic translation initiation factor 5A2 activation. Cancer Sci 105: 219-227, 2014.

35. Yang H, Li XD, Zhou Y, Ban X, Zeng TT, Li L, Zhang BZ, Yun J, Xie D, Guan XY and Li Y: Stemness and chemotherapeutic drug resistance induced by EIF5A2 overexpression in esophageal squamous cell carcinoma. Oncotarget 6: 26079-26089, 2015. 